\title{
SPACE SHUTTLE AND SPACE STATION RADIO FREQUENCY (RF) EXPOSURE ANALYSIS
}

\author{
Shian U. Hwu, Barrios Technology, and Yin-Chung Loh, Jacobs Engineering, Houston, TX \\ Catherine C. Sham, and Quin D. Kroll, NASA Johnson Space Center, Houston, TX
}

\begin{abstract}
This paper outlines the modeling techniques and important parameters to define a rigorous but practical procedure that can verify the compliance of RF exposure to the NASA standards for astronauts and electronic equipment. The electromagnetic modeling techniques are applied to analyze RF exposure in Space Shuttle and Space Station environments with reasonable computing time and resources. The modeling techniques are capable of taking into account the field interactions with Space Shuttle and Space Station structures. The obtained results illustrate the multipath effects due to the presence of the space vehicle structures. It's necessary to include the field interactions with the space vehicle in the analysis for an accurate assessment of the RF exposure. Based on the obtained results, the RF keep out zones are identified for appropriate operational scenarios, flight rules and necessary RF transmitter constraints to ensure a safe operating environment and mission success.
\end{abstract}

\section{Introduction}

Radio Frequency (RF) exposure analysis is important for Space Shuttle Orbiter (SSO) and International Space Station (ISS) mission planning and operations. There are concerns about the potential RF hazard to astronauts and equipment during the operations of the SSO and ISS high power transmitters. The Space Station Orbital Replacement Units (ORUs) and Space Shuttle avionics are designed to meet specific electromagnetic susceptibility requirements. Excessive RF exposure can risk astronaut health; degrade RF system performance; cause equipment malfunctions; and damage the sensitive electronic circuits and components. To prevent potential RF hazards to astronauts and equipment, constraints on the RF transmitters operations may be necessary during missions.

Figure 1 shows the flight configuration of Space Shuttle docked to Space Station. As can be seen, the Station and Shuttle vehicle structures will affect the field intensity and distribution around the transmitters. When the Shuttle is docked to the Space Station, the multiple reflections from both vehicle structures can significantly alter the antenna pattern in near field range. The field strength and distributions are altered due to constructive and destructive interferences among direct and reflected fields.

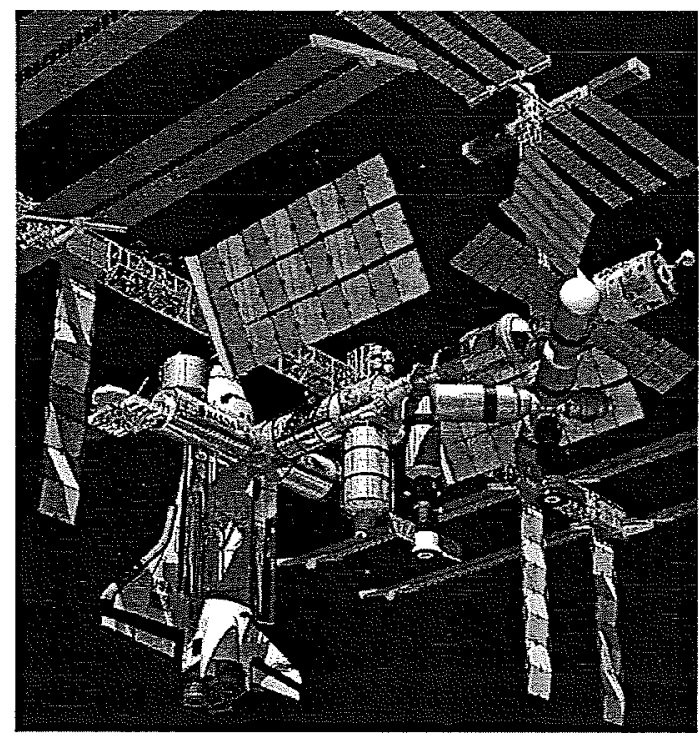

Figure 1. Shuttle Docked to Space Station

Antenna anechoic chamber measurements and/or electromagnetic modeling tools can be used for the RF exposure analysis. Antenna anechoic chamber measurements for Space Shuttle and Space Station are very time consuming and difficult. In some operational scenarios, it may not be possible to carry out due to the large size and complex structure of the space vehicle. For this reason the 
electromagnetic modeling tools are the preferred method for the on-orbit RF exposure analysis.

Two rigorous but practical computer simulation methods for RF exposure assessments are proposed and applied for selective Space Shuttle and Space Station transmitters. Since the reflections from the surrounding structures can significantly alter and increase the field level with respect to the free space case, the multipath effects have to be taken into account for the accuracy of the field calculations.

The electromagnetic field reflections and diffractions by the Space Shuttle and Space Station structure can be computed using the rigorous electromagnetic modeling techniques. The electric fields around the vehicles were computed including the reflections and diffractions from the SSO and ISS structures. The total field levels are computed by summing up the radiation from the transmitting antenna and interactions from the surrounding vehicle structures.

The RF exposure analysis for safety is similar to the RF coverage analysis for communication performance. Both require computing or measuring the intensity of the field in a region around the transmitters. The RF coverage analysis determines the coverage area with the field intensity greater than the required minimum threshold level based on the sensitivity of the receiver. The RF exposure analysis determines the hazard area with the field intensity greater than the specified maximum permissible exposure limit based on the susceptibility of the equipment or the risk of human health. As a conservative approach, maximum transmit power, maximum antenna efficiency and minimum system loss should be used for the RF exposure analysis. On the other hand, minimum transmit power, minimum antenna efficiency and maximum system loss are typically used for the RF coverage analysis.

To obtain a general solution valid in both the near-field and the far-field, the electric and magnetic fields of each radiating element in the antenna were calculated at grid points and a vector summation was performed. The total electric field densities at grid points were computed from the vector summation of directed, reflected, and diffracted fields. Two- and three-dimensional field density maps were generated.
Computer simulations yielded a map of the RF field strength in selected areas. By comparing the computed field strength to corresponding RF exposure limit specifications, the risks to human and certain equipment can be assessed. Assessing the risks led to recommending the appropriate operational scenarios, flight rules and necessary RF transmitter constraints to ensure a safe operating environment and mission success.

\section{COMPUTATIONAL METHODS}

This section presents the proposed modeling techniques to quantify and map the $R F$ radiation intensity around Space Shuttle and Space Station RF transmitters. Computational analyses were performed using the proposed electromagnetic modeling techniques taking into account the field interactions from the Space Shuttle and Space Station structures. Based on the results obtained, the $\mathrm{RF}$ exposure keep out zones for equipment and astronauts were determined for developing appropriate flight rules.

The Space Shuttle and Space Station structure are electrically large in terms of wavelength at frequencies above UHF band. The Geometrical Theory of Diffraction method (GTD) is a suitable candidate for the computational task [1-4]. The Method of Moments (MoM) is a feasible tool and provides better accuracy for electrically small payloads and partial vehicles [5-7]. Excessive computer resources and computing time will be required for electrically large models using the MoM technique.

\section{Geometrical Theory of Diffractions (GTD)}

The Geometrical Theory of Diffractions [1-4] was used in the simulations to take into account the multipath and blockage effects from the Space Shuttle vehicle structures. This method is applicable for electrically large objects which are larger than a few wavelengths.

At high frequencies the scattering fields depend on the electrical and geometrical properties of the scatterer in the immediate neighborhood of the point of reflection and diffraction. In the field computation, the incident, reflected, and diffracted fields are determined by the field incident on the 
reflection or diffraction point multiplied by a dyadic reflection or diffraction coefficient, a spreading factor, and a phase term. The reflected and diffracted field at a field point $\mathrm{r}^{\prime}, E^{\mathrm{r}, \mathrm{d}}\left(\mathrm{r}^{\prime}\right)$, in general have the following form:

$$
E^{\mathrm{r}, \mathrm{d}}\left(\mathrm{r}^{\prime}\right)=E^{\mathrm{i}}(\mathrm{r}) D^{\mathrm{r}, \mathrm{d}} A^{\mathrm{r}, \mathrm{d}}(\mathrm{s}) \mathrm{e}^{-\mathrm{jks}} .
$$

where $E^{i}(r)$ is the field incident on the reflection or diffraction point $\mathbf{r}, D^{\mathrm{rd}}$ is a dyadic reflection $\left(\boldsymbol{D}^{\mathrm{r}}\right)$ or diffraction $\left(D^{\mathrm{d}}\right)$ coefficient, $A^{\mathrm{r}, \mathrm{d}}(\mathrm{s})$ is a spreading factor for reflection or diffraction, and $s$ is the distance from the reflection or diffraction point $\mathrm{r}$ to the field point r'. $D^{\mathrm{r}, \mathrm{d}}$ and $A^{\mathrm{r}, \mathrm{d}}$ can be found from the geometry of the structure at reflection or diffraction point $r$ and the properties of the incident wave there. Thus, the total fields $\left(\mathrm{E}^{\text {tot }}\right)$ can be obtained by summing up the individual contributions of the direct field $\left(E^{\mathrm{dir}}\right)$, reflected field $\left(\mathrm{E}^{\mathrm{ref}}\right)$, and diffracted field $\left(\mathrm{E}^{\mathrm{dif}}\right)$ along the propagation paths, as shown in Fig. 2,

$$
E^{t o t}=E^{d i r}+\sum_{n=1}^{N} E_{n}^{r e f}+\sum_{m=1}^{M} E_{m}^{d i f} .
$$

$E^{\text {tot }}$ : Total field at the observation point,

$E^{\text {dir }}$ : Direct fields from antennas,

$\mathrm{E}^{\text {ref }}$ : Reflected fields from plates and curve surfaces,

$\mathrm{E}^{\mathrm{dif}}:$ Diffracted fields from plates and curve surfaces,

where $\mathrm{n}$ is the $\mathrm{n}^{\text {th }}$ reflection and $\mathrm{N}$ is the total number of reflections. The $m$ is the $\mathrm{m}^{\text {th }}$ diffraction and $M$ is the total number of diffractions.

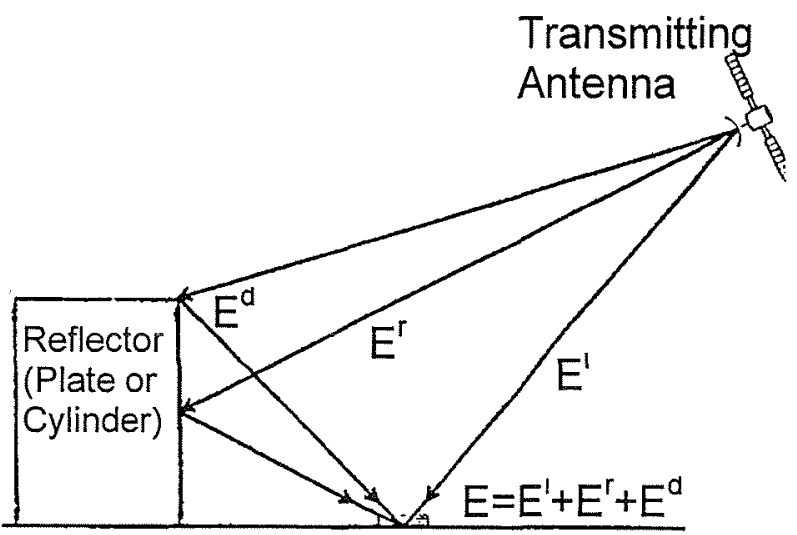

Figure 2. The GTD field computation.

\section{Method of Moments (MoM)}

A rigorous modeling technique to compute the near field strengths for an antenna mounted on an arbitrary structure has been developed [5-7] for electrically small objects which are not larger than a few wavelengths. The electric field integral equation (EFIE) is formulated in the frequency domain using the vector and scalar potential description for an arbitrarily shaped, perfectly conducting structure consisting of surfaces and wires. The surfaces are modeled by planar triangular patches, and thin wires are modeled by piecewise linear segments. The integral equations are formulated via the equivalence principle, and the method of moments is applied to solve for the currents induced on the boundary surfaces of the system. The current distribution on the structure can be obtained for each specified excitation by solving the resulting matrix equation. From these currents, the near field can be obtained.

Let $\mathrm{S}$ denote a configuration of an antenna immersed in an incident electromagnetic field. In general, $S$ may consist of a collection of conducting bodies $\mathrm{S}_{\mathrm{B}}$ and wires $\mathrm{S}_{\mathrm{W}}$. An electric field $\boldsymbol{E}^{i}$, defined to be the field due to an impressed source in the absence of $\mathrm{S}$, is incident on and induces surface current $J$ and total current $I$ on $S_{B}$ and $S_{W}$ respectively. A pair of coupled integral equations for the configuration of wires and bodies may be derived by requiring the tangential component of the electric field to vanish on each surface. Thus we have

$$
E_{t a n}^{i}=(j \omega A+\nabla \Phi)_{t a n}
$$

Where

$$
\begin{aligned}
& A=\frac{\mu}{4 \pi}\left(\int_{S_{B}} J \frac{e^{-j k R}}{R} d S+\int_{S_{W}} \frac{I}{2 \pi a\left(s^{\prime}\right)} \frac{e^{-j k R}}{R} d S\right)(4) \\
& \Phi=\frac{j}{4 \pi \omega \alpha}\left(\int_{S_{B}} \nabla \cdot J \frac{e^{-j k R}}{R} d S+\int_{S_{W}} \frac{1}{2 \pi x\left(s^{\prime}\right)} \frac{d I}{d s} \frac{e^{-j k R}}{R} d S\right)(5)
\end{aligned}
$$

and $\boldsymbol{R}=\left|\boldsymbol{r}-\boldsymbol{r}^{\prime}\right|$ is the distance between an arbitrarily located observation point $r$ and a source point $r$ ' on $S$. In (4) and (5), the wavenumber 
$k=2 \pi / \lambda$, where $\lambda$ is the wavelength, $\varepsilon$ and $\mu$ are the permittivity and permeability, respectively, of the surrounding medium, $\boldsymbol{s}^{\prime}$ is the arc length along the wire axis, and $a$ is the radius of the wire.

The current is written as a linear combination of subdomain basis functions with unknown expansion coefficients. These unknown coefficients are obtained by applying the method of moments for solving the integral equation.

The vector and scalar potentials $\boldsymbol{A}$ and $\Phi$ can be calculated at any point in space according to the formulas (4) and (5). Finite differences were used to approximate the gradient and curl operations, and hence to determine the near electric and magnetic fields at a given point. The advantages of this method are (1) that the multipath interaction effects between antenna and mounting structure are included and (2) it's capable of both far-field and near-field predictions.

Note that the computation time for electrically large objects using MoM can be excessive. The induced currents are computed by solving a system of linear equations of order $\mathrm{N}$, where $\mathrm{N}$ is the total number of unknown currents. The computation time for solving the equation system is of order $\mathrm{N}^{3}$ by using the Gauss-elimination method. For surface currents, the number of unknowns is proportional to the square of frequency $f^{2}$. The computation time is of order $\mathrm{f}^{6}$. For electrically large objects, the required computing time and computer memory can be excessive and out of the hardware capabilities.

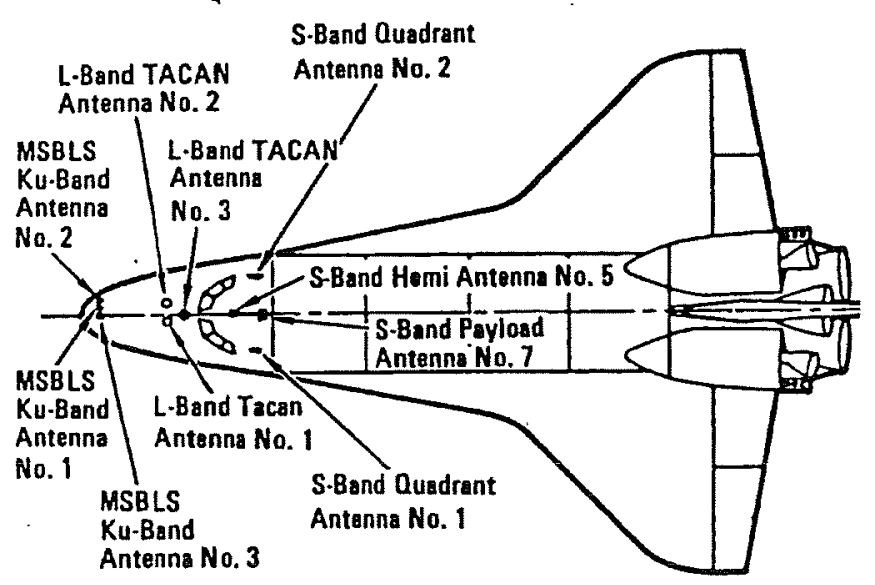

Figure 3. Shuttle TACAN antenna locations.

\section{Space Shuttle Transmitters}

There are many transmitters on the Space Shuttle, as shown in Fig. 3. The transmitters with high power radiating capacity such as the Standard Tactical Air Navigation (TACAN) antennas are of particular concern to the RF exposure analyzer. The UHF antenna inside the payload bay is also a concern since many payloads and avionics are located in payload bay. The GTD technique was applied for the field computations due to the high frequency transmitters and large vehicle structure interactions.

\section{Space Shuttle TACAN Antennas}

The SSO TACAN transmitter has a nominal power output of $1.2 \mathrm{KW}$. The antennas are annular slots with peak antenna gain about $3 \mathrm{~dB}$. The Space Shuttle and Space Station robotic arm or Remote Manipulator System (RMS) will be operated very close to the TACAN antennas during Orbiter underside thermal tile inspection and repairing operations. The electric field can be very high, exceeding hundreds of Volts per meter, in close proximity of the TACAN antennas. These high level electric fields can be hazardous to crewmen health and sensitive electronic equipment around the transmitter. For mission planning, the RF keep out zone has to be determined and analyzed for appropriate flight rule development.

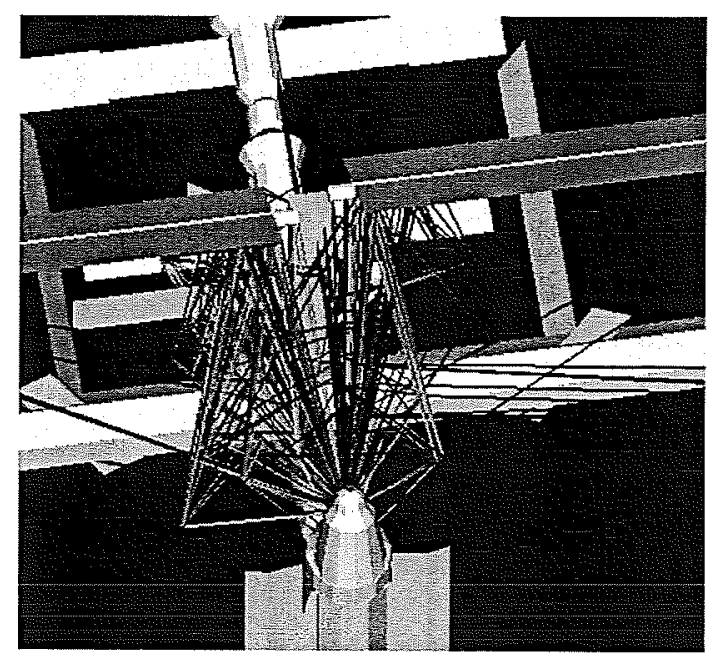

Figure 4. Ray tracing illustrating structure interactions for the Shuttle TACAN antenna.

Figure 4 shows ray tracing for the TACAN transmitter on the Space Shuttle. As illustrated, 
many bounces of reflections from the surrounding Space Station structures exist. As a result, the antenna pattern and thus the field strength and distributions will be affected significantly due to constructive and destructive interferences among direct and reflected fields.

Since the TACAN antennas will only be radiating while on-orbit for testing, the robotic arms should be moved outside the RF Keep Out Zone (KOZ) from the TACAN antenna during the TACAN system testing. The KOZ is based on electronic equipment radiated susceptibility limit and represents the minimum required distance between the radiating antenna and other equipment to avoid off-nominal operation of the equipment due to excessive RF exposure.

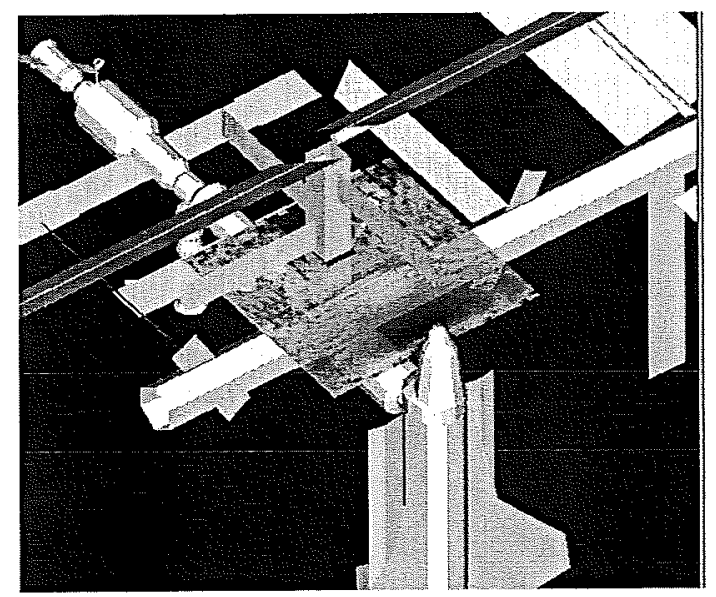

Figure 5. Computed field in a horizontal plane due to the Shuttle TACAN antenna.

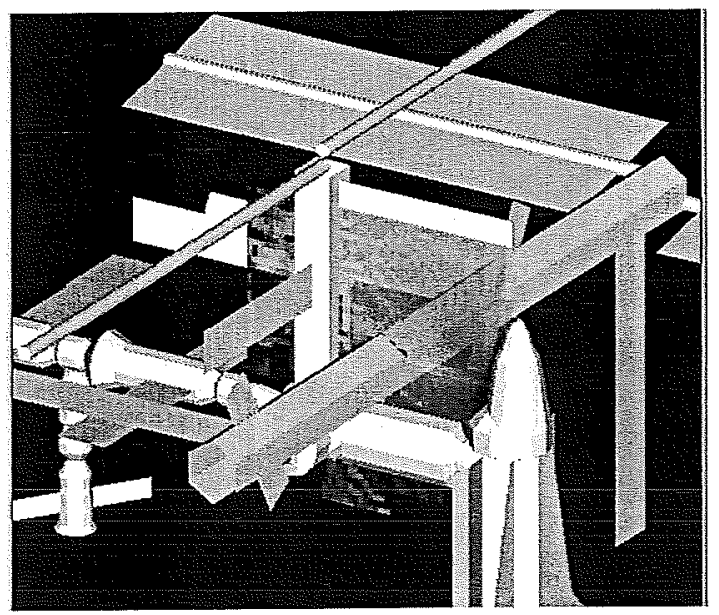

Figure 6. Computed field in a vertical plane due to the Shuttle TACAN antenna.

\section{Space Shuttle UHF Antenna}

The Space Shuttle payload bay Ultra High Frequency (UHF) antenna is designed to provide communication services to the Extravehicular Activity (EVA) astronauts. Since many payloads and avionics are located in payload bay, the field intensities in payload bay due to the UHF antenna radiation have to be quantified for the RF safety analysis.

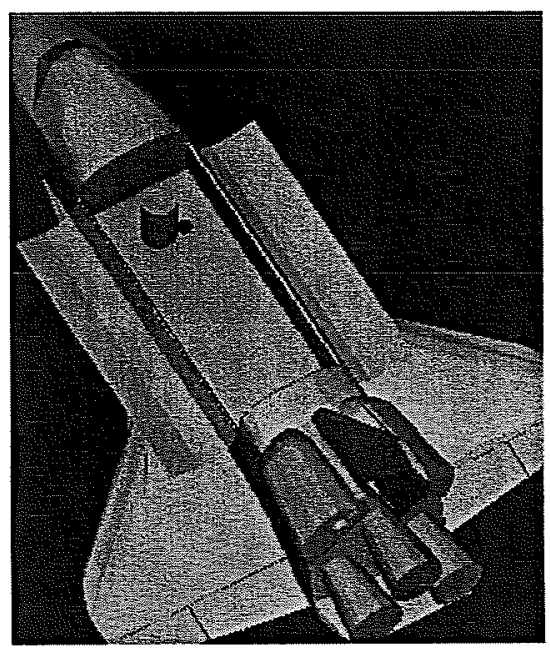

Figure 7. Shuttle UHF antenna location (in red dot). The near-by cylinder is the Orbiter docking system.

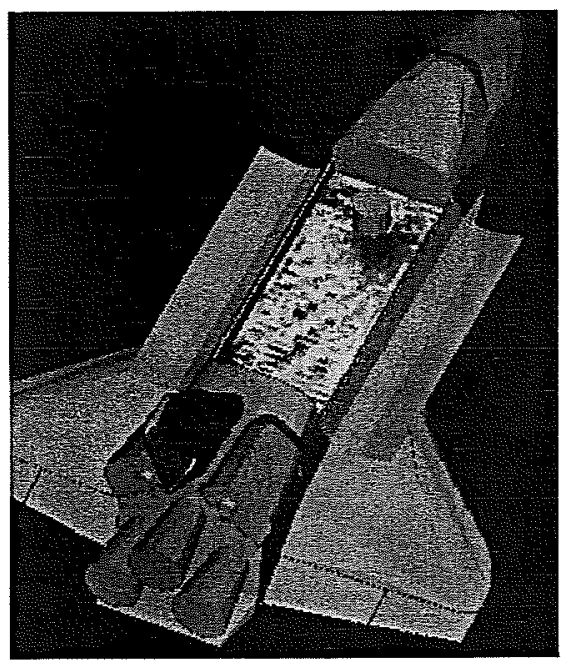

Figure 8. Computed field in payload bay due to the Shuttle UHF antenna. High level fields are in red and orange and low level fields are in blue and green.

The Space Shuttle payload bay UHF antenna is a quadrifilar helical antenna located inside the payload bay near the Orbiter Docking Adaptor 
(ODS), as shown in Fig. 7. The peak gain is about $3.5 \mathrm{~dB}$. The computed field map inside the payload bay is shown in Fig. 8. The effects of reflections from the payload bay walls and blockages from the ODS were observed in the forms of peaks and valleys in the computed field distributions inside the payload bay.

\section{Space Station Transmitters}

There are many transmitters on the Space Station. To demonstrate the applicability of the MoM technique for electrically small object modeling, a payload transmitter was selected for the $\mathrm{RF}$ exposure analysis. The MoM technique was applied for the field computations due to the lower frequency transmitter and smaller payload structure interactions. Note that the accurate MoM model requires detail mechanical dimensions of the antenna and attached structures.

\section{Space Station MISSE Antennas}

The Materials International Space Station Experiment (MISSE) is a payload on the Space Station. The purpose of MISSE payload is to characterize the performance of new prospective spacecraft materials when subjected to the synergistic effects of the space environment. Figures 9 and 10 show the various MISSE installation sites on the Space Station.

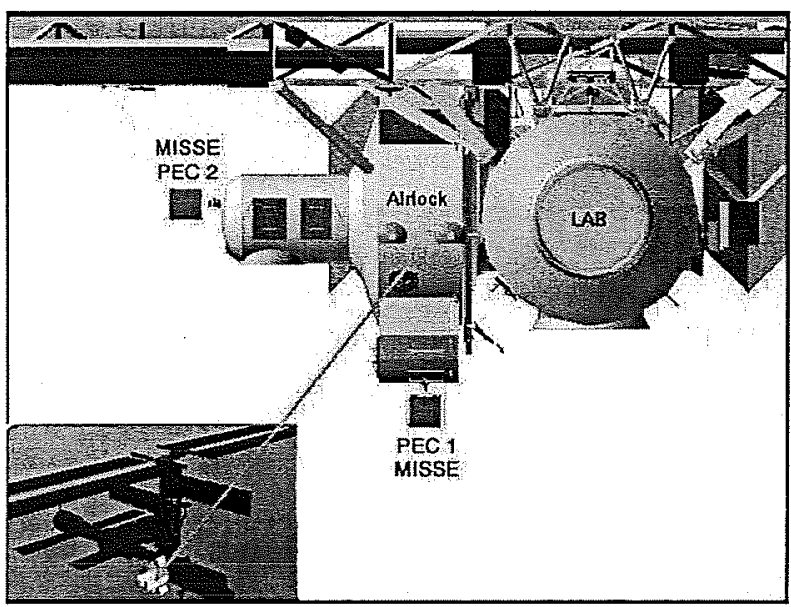

Figure 9. The planned various MISSE installation sites on Space Station.

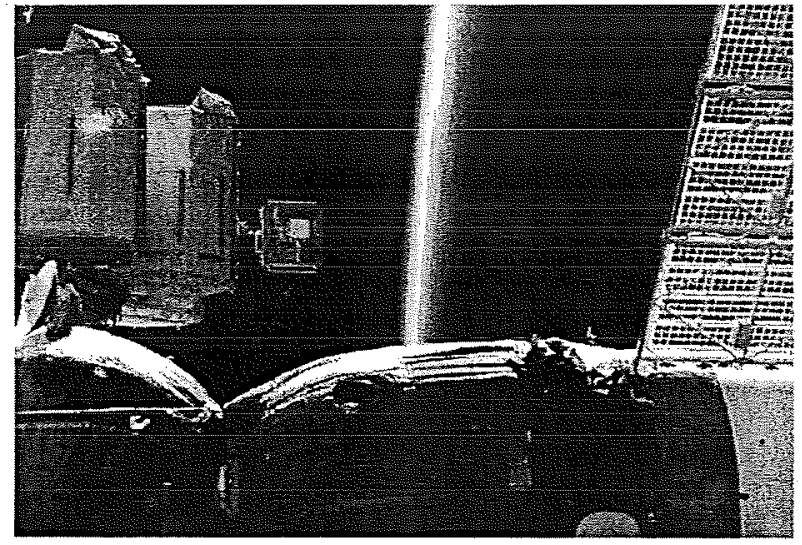

Figure 10. The previously installed MISSE on Space Station.

It is difficult to estimate the near-field strengths within a very close range of the antenna mounted on an arbitrary structure. When the range is within the near field, the simple far field formula is no longer valid and hence rigorous modeling techniques [5-7] have to be applied. In order is to quantify the electromagnetic field intensity around the Space Station MISSE antennas, the MoM technique was used to model both antennas and attached MISSE payload structures.

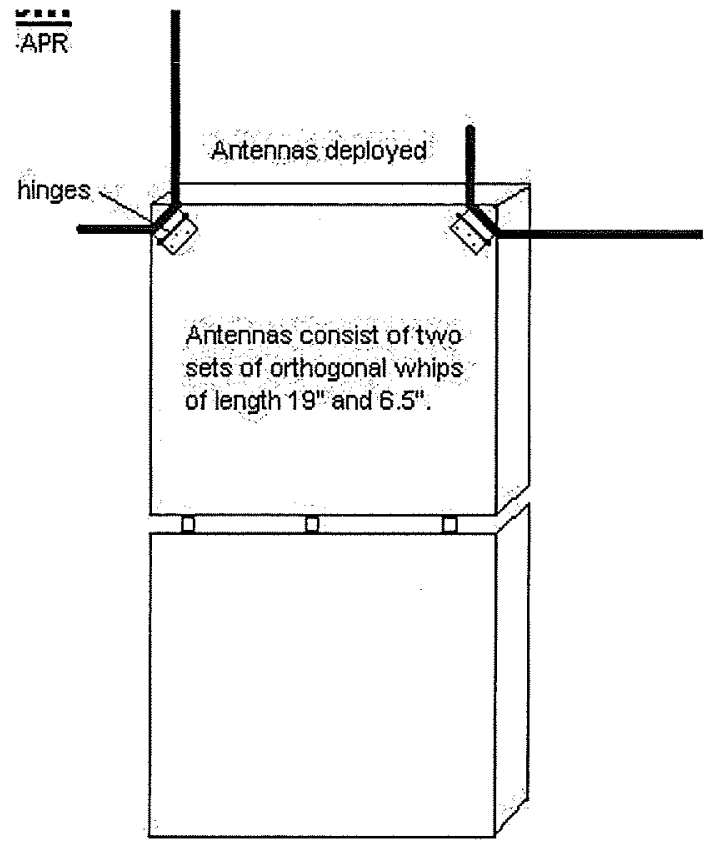

Figure 11. The MISSE dual pair antenna configuration.

The MISSE antenna configuration is shown in Fig. 11. The MISSE payload has two pairs of monopole antennas. Each pair of antennas is 
oriented orthogonal and radiated simultaneously to provide polarization diversity communication coverage from the ground. The 19" monopole antenna pair is operated at frequency of $145.8 \mathrm{MHz}$. The 6.5 " monopole antenna pair is operated at frequency of $436.8 \mathrm{MHz}$.

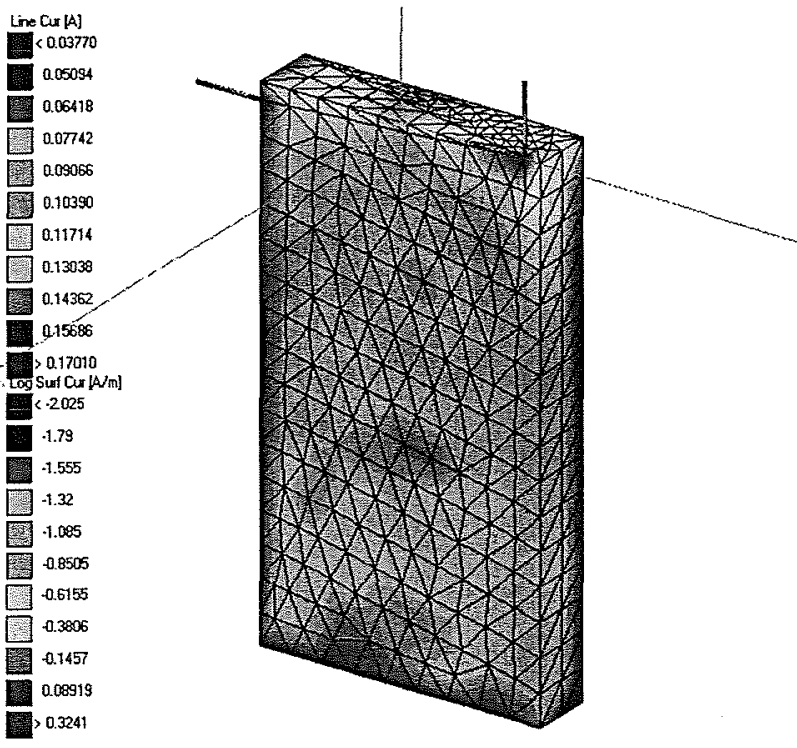

Figure 12. Induced currents from the 436.8 MHz pair MISSE antenna.

The transmit power for each antenna is $1 \mathrm{Watt}$. The total power for each pair of antennas is 2 Watts. The minimum circuit loss is assumed to be 0 $\mathrm{dB}$ as the worst case scenario. Dual orthogonal antenna configuration for each frequency was analyzed. The method of moments presented in the previous section [5-7] was applied for the induced currents and near-field computations. The induced currents on the MISSE antennas and payload are shown in Figs. 12 and 13. As expected, the hot spots are near the base of the quarter wave monopole antennas. The computed near-fields around the MISSE antennas are shown in the Figures 14 and 15. The near-field distributions close to the antenna are quite different for the two different pair MISSE antennas due to the structure interactions from the attached MISSE payload structure. Note that the transmit power and antenna gain for the two pair antennas are almost identical except operated at two different frequencies.

Based on the results obtained, the RF exposure keep-out distances were determined for the MISSE antennas.

\section{Conclusion}

Rigorous but practical electromagnetic modeling techniques were employed to quantify the near fields around the Space Shuttle and Space Station RF transmitters. The GTD technique is suitable for the field computations from the higher frequency transmitters with larger space vehicle structure interactions. The MoM technique is applicable for the field computations from the lower frequency transmitters with smaller space vehicle structure interactions. The computed fields include multipath effects from the surrounding Space Shuttle and Space Station structures.

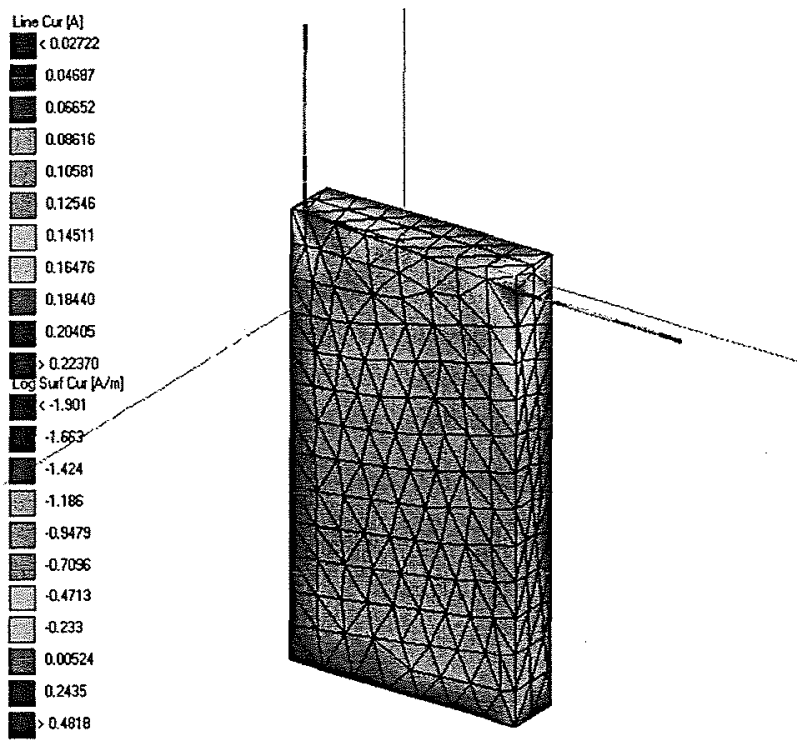

Figure 13. Induced currents from the $\mathbf{1 4 5 . 8}$ MHz pair MISSE antenna.

The applicability of the proposed electromagnetic modeling techniques was demonstrated for selected Space Shuttle and Space Station transmitters. These modeling tools can significantly reduce the time consuming and costly field measurements. Based on the results obtained, the astronaut and equipment RF exposure safety can be analyzed. For example, software masks were implemented to prevent the pyrotechnic devices illuminated by the steerable high gain antenna. This RF field mapping data for high power transmitters is important for developing Shuttle and Space Station transmitters' operational constraints and flight rules. 


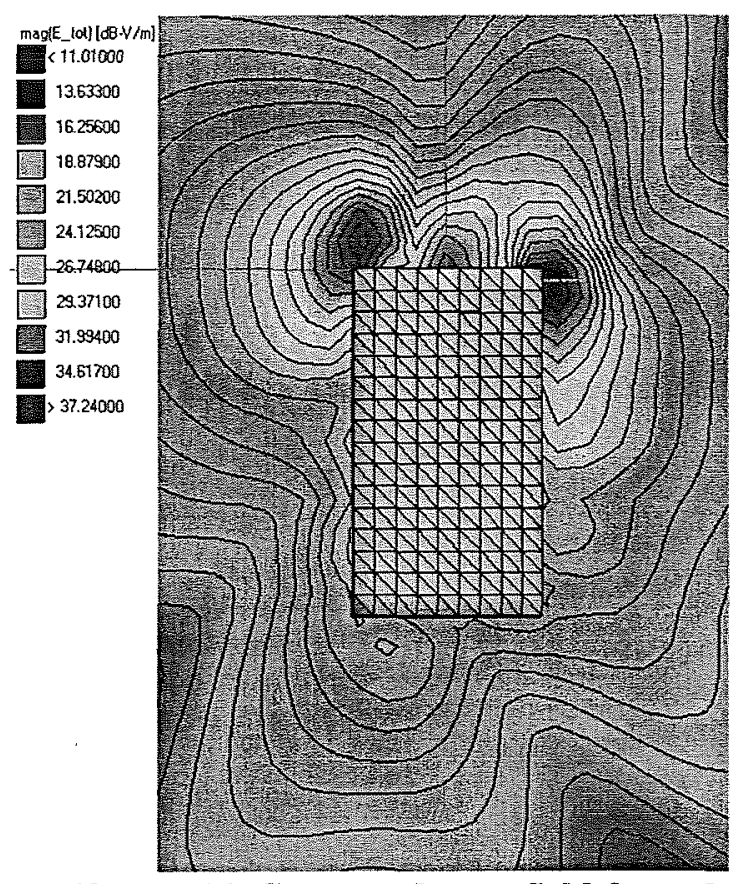

Figure 14. Computed near-field from the 436.8 MHz pair MISSE antenna.

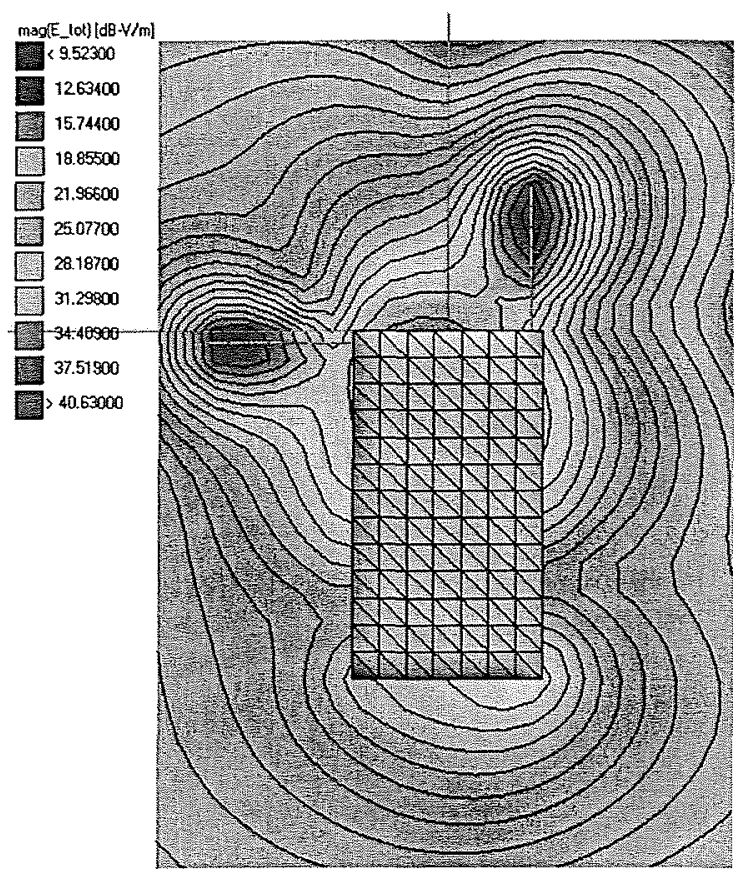

Figure 15. Computed near-field from the 145.8 MHz pair MISSE antenna.

\section{References}

[1] Marhefka, R.J., and Silvestro, J.W., "Near zone - basic scattering code user's manual with space station applications," NASA CR-181944, Dec. 1989.
[2] Marhefka, R.J. and Burnside, W.D. "Antennas on complex platforms," Proceedings of the IEEE, Volume: 80, Issue: 1, pp. 204-208, Jan. 1992.

[3] Marhefka, R.J. "The other NEC (BSC) an asymptotic complement," Antennas and Propagation Society Symposium, 2004. IEEE, Vol.3, pp. 2911 -2914, 20-25 June 2004.

[4] Hwu, S.U., Loh, Y.C., Sham, C.C., and Kroll, Q.D., "International space station Ku-band communications antenna blockage analysis," Journal of spacecraft and Rockets, pp. 232-233, Vol. 41, No. 2, Mar. 2004.

[5] Hwu, S.U.; Wilton, D.R.; Rao, S.M., "Electromagnetic scattering and radiation by arbitrary conducting wire/surface configurations," Antennas and Propagation Society International Symposium, 1988. AP-S. Digest, Syracuse, NY, 610 June 1988 Page(s):890 - 893 vol. 2

[6] Sharpe, R.M.; Grant, J.B.; Champagne, N.J.; Johnson, W.A.; Jorgenson, R.E.; Wilton, D.R.; Brown, W.J.; Rockway, J.W., "EIGER: Electromagnetic Interactions GEneRalized," Antennas and Propagation Society International Symposium, 1997. IEEE, 1997 Digest, Volume 4, 13-18 July 1997 Page(s):2366 - 2369 vol.4

[7] S. Hwu, J. Fournet, D. Eggers, G. Arndt," Space Shuttle EMI Analysis : Electromagnetic Field Strength Around WISP Payload," Proc. 1992 IEEE International Electromagnetic Compatibility Symposium, pp. 219-222, Anaheim, CA, August 1992.

\section{Acknowledgment}

This work was supported in part by the NASA Johnson Space Center under ESC Contract.

The authors wish to thank the contributions from John P. Boster of Jacobs Engineering and technical review and comments by Laura Hood of NASA/JSC.

\section{Email Addresses}

Shian U. Hwu (shian.u.hwu1@jsc.nasa.gov). Yin-Chung Loh (yin.loh@escg.jacobs.com). Catherine C. Sham (catherine.c.sham@nasa.gov). Quin D. Kroll (quin.kroll-1@nasa.gov). 
$24^{\text {th }}$ Digital Avionics Systems Conference October 30, 2005 